\title{
Comunicación

\section{Situación profesional y}

\section{satisfacción laboral de los}

\section{periodistas colombianos}

\section{Professional situation and job satisfaction of}

Colombian journalists

\section{LILIANA GUTIÉRREZ-COBA ${ }^{1}$}

DOI: https://doi.org/10.32870/cys.v2020.7556

Este artículo expone las características laborales de los periodistas colombianos, su nivel de satisfacción con sus condiciones de trabajo y las variables sociodemográficas asociadas. A través de 300 encuestas y 40 entrevistas en profundidad, los resultados muestran un empeoramiento de las condiciones salariales en los últimos diez años, aunque una mejora en las modalidades de contratación. La percepción global de la satisfacción laboral de los periodistas es moderada y existen factores extrínsecos/intrínsecos asociados, como la falta de programas de capacitación y de oportunidades de promoción.

PALABRAS ClAVE: Periodista, condiciones laborales, satisfacción laboral, Colombia.

This article exposes labor characteristics of Colombian journalists, their satisfaction level with their work conditions, and socio-demographic variables associated. Through 300 surveys and 40 in-depth interviews, results show a worsening of salary conditions in the last ten years, although an improvement in hiring modalities. Journalists' global job satisfaction perception is moderate and there are extrinsic/intrinsic factors associated such as lack of training programs and promotion opportunities.

KEYWORDS: Journalist, working conditions, job satisfaction, Colombia.

Cómo citar este artículo:

Gutiérrez-Coba, L. (2020). Situación profesional y satisfacción laboral de los periodistas colombianos. Comunicación y Sociedad, e7556. https://doi.org/10.32870/cys. v2020.7556

Esta investigación fue financiada por la Convocatoria Interna de la Universidad de La Sabana, COM712016.

1 Universidad de La Sabana, Colombia.

liliana.gutierrez1@unisabana.edu.co

Fecha de recepción: 16/07/19. Aceptación: 04/02/20. Publicado: 17/06/20. 
El interés por garantizar que la información que llega a la sociedad sea verdadera, útil y bien elaborada ha tomado fuerza en los últimos años, aun cuando el concepto de calidad informativa o periodística tiene distintas acepciones, los autores suelen concordar en que envuelve las distintas fases de producción de contenidos informativos: la consulta de las fuentes, la elaboración del producto y su posterior distribución (Gómez-Mompart et al., 2013; Gutiérrez-Coba, 2009; Schulz, 2000).

También algunos autores concuerdan en que existen diversos condicionantes para que los procesos periodísticos se den de la manera adecuada. Por una parte, hay condicionantes externos, como la existencia de disposiciones legales que garanticen la libertad de prensa y medidas de protección para los periodistas que ejercen su profesión en lugares inmersos en conflictos armados o contextos de inseguridad (Espino, 2016; Garcés \& Arroyave, 2017). En el caso de Colombia, las condiciones de violencia generadas por guerrilla, paramilitarismo y delincuencia organizada han dificultado el ejercicio periodístico en diversas regiones del país. La Fundación para la Libertad de Prensa (FLIP, 2018), señalaba en su informe de 2018 que la cifra de ataques a la prensa tuvo un incremento del 53\% con relación al 2017 y del 120\% con relación al año anterior. Secuestros, hostigamientos, amenazas, obstrucciones a la prensa, eliminaciones de material periodístico, acoso judicial, violencia de género, exilios y desplazamientos internos siguen siendo problemas a los que se deben enfrentar los periodistas colombianos.

También, acorde a los investigadores en la calidad periodística, deben existir regulaciones que eviten la concentración de los medios en pocas manos, de manera que se garantice la diversidad de miradas sobre la realidad, pues la estructura empresarial de los medios y los grupos de poder asociados, tiene una importante influencia en la independencia de los periodistas para realizar su trabajo, como ha sido documentado a nivel latinoamericano (Becerra \& Mastrini, 2017; Huerta-Wong \& García, 2013; Trejo, 2010) e igualmente en Colombia (Consejo de Redacción \& Poderopedia, 2015; Federación Colombiana de Periodistas [Felcoper] \& Reporteros Sin Fronteras, s. f.). En el caso colombiano, la propiedad del $57 \%$ de los medios de comunicación se concentra en tres grupos económicos: Organización Luís Carlos Sarmiento Angulo, Organización Ardila Lulle y Valorem. También 
existen tres canales públicos de televisión que son propiedad del gobierno -Señal Colombia, Canal Institucional y Canal UNO-, siete departamentos 2 del país tienen un canal regional, y tres de ellos canales locales de propiedad de los entes territoriales donde se encuentran ubicados y cuya financiación en buena parte es de la Autoridad Nacional de Televisión (ANTV) y de los gobiernos departamentales o municipales. Igualmente, hay medios comunitarios, es decir, medios sin ánimo de lucro, creados y manejados por las propias comunidades en barrios y localidades de distintos municipios del país, los cuales suelen funcionar en condiciones precarias.

A lo anterior se suma una gran variedad de emprendimientos nativos digitales, de los cuales para 2012 se contaban 88 (Rey \& Novoa, 2012), pero que han proliferado en las últimas décadas, de manera que para 2018 se registraban 334 (Barragán, 2018). Sin embargo, el más reciente informe trimestral del Ministerio de Tecnologías de la Información y las Comunicaciones (2019) señala que al finalizar el cuarto trimestre de 2018 el país alcanzó un total de 32.7 millones de conexiones a Internet de banda ancha, lo cual representa un índice de penetración de $65.5 \%$, mientras estadísticas de la International Telecommunication Union (2018), mostraban que para 2017, Colombia se encontraba en la posición 19 entre 27 países de América Latina en cuanto a la penetración de Internet móvil (48.8 conexiones activas por cada 100 habitantes), lo cual muestra un rezago del país en materia de conectividad, que termina por influir en el consumo de medios digitales.

Otras condicionantes son más propias del fuero interno del periodista, como el cumplimiento de estándares de verificación de información, rutinas de reportería adecuadas y la adhesión a compromisos de carácter ético con la verdad y la independencia (Casero-Ripollés \& Rabadán, 2013; Pellegrini et al., 2011; Reyes et al., 2015; Romero-Rodríguez et al., 2016).

Finalmente, hay condicionantes que dependen de la empresa periodística, que debe garantizar los espacios y recursos necesarios para que los periodistas puedan realizar bien sus tareas, ambientes de trabajo saludables y condiciones laborales justas, que incluyen una contratación

2 Estados o Comunidades. 
que implique estabilidad y salarios dignos (Mellado \& Lagos, 2013; Ufarte, 2012).

La conjunción de todos estos factores permitiría garantizar que los ciudadanos reciban información de buena calidad, que sirva al bien común y no a los intereses de unos pocos.

Como se observa, la calidad periodística resulta un fenómeno complejo, de manera que los investigadores suelen abordar por separado algunos de estos aspectos, en este caso, las condiciones laborales y la satisfacción de los periodistas con su trabajo, en el entendido de que sin salarios justos y en situaciones de precariedad, la motivación y el compromiso con los valores periodísticos pueden verse comprometidos.

\section{Precariedad en la sombra}

En Colombia, los estudios sobre las condiciones laborales de los periodistas son escasos. Menos de un puñado de investigaciones se han hecho sobre el tema en los últimos 15 años (Barrios \& Arroyave, 2007; Gutiérrez-Coba et al., 2010; Manrique \& Cardona, 2003). En 2015, la Fundación para la Libertad de Prensa (FLIP, s. f.) inició un mapeo de los medios de comunicación existentes en Colombia, que es quizá el estudio más exhaustivo que se ha llevado a cabo sobre la realidad del sistema informativo en el país. Aunque todavía los resultados son preliminares porque no han terminado de realizar las entrevistas con representantes de cada uno de los más de dos mil medios encontrados, el estudio da cuenta de un panorama preocupante, de bajos salarios e inexistencia de contratos laborales en los medios ubicados en los municipios más apartados de las capitales.

El trabajo publicado más reciente sobre el tema data del año 2010 (Gutiérrez-Coba et al., 2010), lo cual muestra el poco seguimiento que han tenido las condiciones laborales de los periodistas y hace pertinente la comparación de ambos estudios, aun cuando no todas las variables sean iguales, para establecer si existe o no mejoría en la situación de trabajo de los periodistas colombianos.

De los anteriores referentes surge la idea de realizar esta investigación, que busca establecer cuáles son las condiciones de trabajo de los periodistas en Colombia, no solamente en términos del tipo de contratación y de cuánto dinero reciben por su labor, sino de otros factores 
como la autonomía, la capacitación, los recursos y los estímulos que se les otorgan para realizar bien su trabajo, todo lo cual incide en su percepción de satisfacción laboral. De tal suerte, las preguntas de investigación que guían este estudio son cuatro: ¿Cuál es el perfil del periodista colombiano?; ¿cuáles son las condiciones laborales de los periodistas colombianos?; ¿qué asociaciones se dan entre el perfil del periodista y mejores condiciones de trabajo?; ¿qué tan satisfechos se sienten los periodistas colombianos con su situación laboral? De esta manera se podrán establecer los puntos específicos en los cuales los gremios de periodistas, las Facultades de Comunicación Social-Periodismo, las empresas periodísticas y el Gobierno, deben trabajar en pro de mejorar la situación laboral de los profesionales de la información.

Lo anterior resulta de especial interés en un contexto en que el periodismo es radicalmente diferente de lo que era a principios de siglo. El incesante desarrollo de las Tecnologías de la Información y la Comunicación ha hecho que los medios se vean en la obligación de replantear su oferta informativa, su estructura empresarial, las relaciones laborales con sus periodistas, la manera en que interactúan con las audiencias y su modelo de negocio. Todo lo cual ha incidido negativamente en las condiciones de trabajo de los informadores, ya que en muchos casos las empresas periodísticas han reducido el número de trabajadores, al tiempo que asumen retos como la convergencia, la instantaneidad y la distribución de contenidos a través de redes sociales; de manera que los periodistas ven cómo aumentan sus tareas mientras se enfrentan también a la incertidumbre laboral que generan las nuevas condiciones de su empleo, tal como lo referencian diversas investigaciones que han constatado cambios en las formas de reportería y distribución de la información, y en las rutinas de trabajo de los periodistas (Barrios \& Zambrano, 2015; Farías \& Gómez, 2011; Márquez-Ramírez \& Hughes, 2017; Mellado \& Lagos, 2013; Odriozola et al., 2016; Sabés \& Verón, 2012; Túñez \& Martínez, 2015).

\section{Contexto sobre la satisfacción laboral}

La satisfacción laboral ha sido tema de estudio de muchos investigadores desde los años sesenta del pasado siglo. Ello ha llevado a definiciones tan sencillas como que se trata del grado en que a los empleados les gusta 
su trabajo (Fisher, 2000; Fritzsche \& Parrish, 2005), lo cual supone una respuesta afectiva o emocional, hasta definiciones más complejas que la presentan como un juicio evaluativo, positivo o negativo que el individuo realiza de su situación de trabajo, y que involucra aspectos como el salario, los horarios, las condiciones físicas del lugar donde labora, entre otros (Bowling et al., 2015; Meyer et al., 2010; Weiss, 2002).

Thompson y Phua (2012) sostienen que la mejor manera de establecer la satisfacción laboral consiste en pensarla en términos de dos categorías diferentes y complementarias. Por una parte, la satisfacción laboral cognitiva, resultado de un proceso de evaluación consciente de las características del trabajo y de su comparación con un parámetro externo $\mathrm{y}$, por otra parte, la satisfacción laboral afectiva, representativa de una respuesta emocional positiva del empleado hacia el trabajo como un todo.

La investigación que presentamos a continuación se identifica con esta última definición, dado que históricamente los estudios sobre la labor periodística han mostrado un contraste paradójico: pese a las condiciones de precariedad en que realizan su trabajo, los periodistas sienten pasión por lo que hacen y su compromiso con la función social sigue intacto (Arroyave \& Blanco, 2005; Cantalapiedra et al., 2016; Díaz Jordán \& Serrano, 2017; Mellado \& Parra, 2008; Rodríguez, 2003).

Así pues, se intentarán identificar los elementos intrínsecos que parten desde la propia percepción del periodista sobre aquellos aspectos de su trabajo que le generan sensación de bienestar, y los elementos extrínsecos, sobre los cuales el periodista no tiene control y que son más objetivos sobre sus condiciones reales de trabajo.

\section{Metodología}

Se realizó un diseño mixto, cuantitativo-cualitativo, compuesto por encuestas y entrevistas semiestructuradas. Dado que no existe un censo fiable sobre el número de periodistas que laboran actualmente en los medios de comunicación colombianos, para la encuesta se tomó como base el mapa de medios realizado por Consejo de Redacción y Poderopedia (2015), que daba cuenta de la existencia de 220 medios de comunicación (88 de prensa, 50 de radio, 44 de Internet y 38 de televisión). Las encuestas fueron enviadas por correo electrónico y Twitter a 500 perio- 
distas, logrando respuesta de 300 de ellos, pertenecientes a 123 medios informativos de 24 ciudades del país, en el periodo comprendido entre febrero de 2018 y febrero de 2019 (ver Tabla 1).

TABLA 1

TIPO DE MEDIO SEGÚN CIUDAD DE PROCEDENCIA

\begin{tabular}{|c|c|c|c|c|c|}
\hline Ciudad & Público & Privado & $\begin{array}{l}\text { Empren- } \\
\text { dimiento }\end{array}$ & $\begin{array}{l}\text { Comu- } \\
\text { nitario } \\
\end{array}$ & Total \\
\hline Arauca (Arauca) & 0 & 0 & 1 & 0 & 1 \\
\hline Armenia (Quindío) & 3 & 0 & 0 & 0 & 3 \\
\hline Barranquilla (Atlántico) & 1 & 5 & 0 & 2 & 8 \\
\hline Bogotá (Cundinamarca) & 20 & 176 & 3 & 5 & 204 \\
\hline Bucaramanga (Santander) & 4 & 5 & 0 & 0 & 9 \\
\hline Cali (Valle) & 4 & 7 & 1 & 0 & 12 \\
\hline Cartagena (Bolívar) & 0 & 2 & 0 & 0 & 2 \\
\hline Cúcuta (Nte. Santander) & 0 & 2 & 0 & 0 & 2 \\
\hline Fusagasugá (Cundinamarca) & 0 & 0 & 1 & 0 & 1 \\
\hline Granada (Antioquia) & 0 & 0 & 0 & 2 & 2 \\
\hline Ibagué (Tolima) & 0 & 4 & 0 & 0 & 4 \\
\hline La Mesa (Cundinamarca) & 0 & 0 & 1 & 0 & 1 \\
\hline Manizáles (Caldas) & 0 & 3 & 0 & 0 & 3 \\
\hline Medellín (Antioquia) & 9 & 11 & 1 & 0 & 21 \\
\hline Montería (Córdoba) & 0 & 2 & 0 & 0 & 2 \\
\hline Neiva (Huila) & 0 & 5 & 0 & 0 & 5 \\
\hline Palmira (Valle) & 0 & 2 & 0 & 0 & 2 \\
\hline Pasto (Nariño) & 1 & 0 & 0 & 0 & 1 \\
\hline Pereira (Risaralda) & 1 & 1 & 0 & 0 & 2 \\
\hline Puerto Berrío (Antioquia) & 0 & 0 & 1 & 0 & 1 \\
\hline Riohacha (Guajira) & 0 & 0 & 1 & 0 & 1 \\
\hline Sopó (Cundinamarca) & 1 & 0 & 0 & 0 & 1 \\
\hline Valledupar (Cesar) & 0 & 2 & 0 & 0 & 2 \\
\hline Villanueva (Guajira) & 0 & 0 & 0 & 1 & 1 \\
\hline Sin reporte & 2 & 4 & 1 & 2 & 9 \\
\hline Total & 46 & 231 & 11 & 12 & 300 \\
\hline
\end{tabular}

Fuente: Datos de la investigación. 
Pese a que se trata de una muestra no probabilística y, por tanto, los resultados no son generalizables a toda la población de periodistas colombianos, estos permiten dar una idea de las condiciones laborales que enfrentan los informadores del país, especialmente aquellos que laboran en medios privados, de quienes se obtuvo mayor número de respuestas.

La encuesta estaba conformada por siete ítems relativos al perfil del periodista, siete sobre condiciones laborales y 11 sobre satisfacción laboral (Alfa de Cronbach 0.906), que se puntúan según una escala Likert entre 1 (Nada satisfecho) y 5 (Muy satisfecho).

El rango de puntuación global del instrumento sobre satisfacción laboral está entre 11 y 55 puntos. Una mayor puntuación se asocia a una mayor percepción global de satisfacción laboral, de manera que se establecieron tres rangos: Satisfacción laboral baja (de 11 a 25 puntos), Satisfacción laboral media (26-40), y Satisfacción laboral alta (41 a 55).

Esta escala está a su vez constituida por dos subescalas. La primera de ellas contempla factores intrínsecos, como el estímulo de sus jefes cuando el periodista hace bien su trabajo, el apoyo que recibe por la misma razón, las oportunidades de ascenso y la autonomía para tomar decisiones (Alfa de Cronbach 0.845). Entre 4 y 9 puntos se considera baja la satisfacción en esta escala, de 10 a 14 puntos satisfacción media y de 15 a 20 satisfacción alta.

La segunda escala atiende factores extrínsecos, es decir, que están fuera del control del trabajador, como el salario, horario, las condiciones físicas y ambientales del lugar de trabajo, las condiciones de salud ocupacional y prevención de accidentes, los programas de capacitación y desarrollo que ofrece la empresa y las actividades de bienestar que ofrece la compañía para la familia del trabajador (Alfa de Cronbach 0.853). La satisfacción extrínseca se considera baja entre 7 y 16 puntos; media entre 17 y 25 puntos, y alta entre 26 y 35 puntos.

Por otra parte, entre mayo y agosto de 2018 , se realizaron entrevistas en profundidad a 40 periodistas de ocho ciudades de Colombia (Bogotá, Cali, Bucaramanga, Medellín, Valledupar, Pereira, Barranquilla y Neiva), buscando precisar algunos aspectos importantes sobre lo que genera satisfacción e insatisfacción de los periodistas frente a su trabajo. Para la selección de los periodistas se tuvo en cuenta que no 
ocuparan cargos directivos en los medios y en cada ciudad se entrevistó a dos periodistas de dos diferentes medios privados, uno público, un emprendimiento y uno comunitario.

\section{RESULTADOS}

\section{El perfil del periodista colombiano y sus condiciones laborales}

El perfil de los 300 periodistas ${ }^{3}$ que respondieron la encuesta está constituido mayoritariamente por hombres (69\%) menores de 40 años de edad $(70.3 \%)$. El rango de edad predominante está entre los 26 y 30 años (22.3\%), seguido del rango entre 31 y 35 años de edad (19.3\%). En porcentajes muy similares, le siguen los que tienen entre 36 y 40 años de edad (14.7\%) y los menores de 25 años (14\%).

En cuanto a la trayectoria profesional, se observan tres bloques: quienes tienen hasta 5 años de experiencia en el periodismo (34\%), los que han laborado entre 6 y 15 años $(32.3 \%)$ y los que se han dedicado entre 16 y más de 20 años al oficio (33\%).

Durante el ejercicio profesional es frecuente el cambio de empleo, de manera que se indagó por la vinculación actual, encontrando que la mayoría trabaja en medios privados $(76 \%)$, seguidos por los medios públicos (16\%), comunitarios (4\%) y emprendimientos (3.7\%). El 34\% de quienes respondieron la encuesta tienen como plataforma principal la televisión, el 29\% los impresos, el 20.3\% la web y el 16.7\% la radio.

El $58.3 \%$ de los encuestados contaba con grado universitario, el $13.7 \%$ con especialización y el $22 \%$ con maestría, lo cual muestra un cambio sustancial al compararlo con lo reportado por Gutiérrez-Coba et al. (2010), donde solo el 5.9\% de los periodistas consultados contaba con estudios de postgrado.

En lo que se refiere a la remuneración salarial, en Colombia el Salario Mínimo Mensual Legal Vigente (SMMLV) para 2019 es de 828 116 pesos, es decir, alrededor de 259 dólares. De los 300 periodistas que respondieron la encuesta, el $26.3 \%$ percibe por su trabajo entre 1.2 y 2.4 SMMLV. Le sigue el $23.3 \%$ que recibe entre 2.4 y 3.6 SMMLV, y

3 Se puede consultar la base de información en https://figshare.com/articles/ DATOS_ENCUESTA_PERIODISTAS_mayo_sav/12269519 
un $10 \%$ que devenga entre 0.6 y 1.2 SMMLV. Incluso, hay un $2.7 \%$ que reportó ganar menos de 0.6 SMMLV. Es decir, que el 62.3\% devenga menos de 3.6 SMMLV, mientras que solo el $37.7 \%$ restante supera este rango (ver Tabla 2).

\section{TABLA 2}

RANGO SALARIAL SEGÚN TIPO DE MEDIO

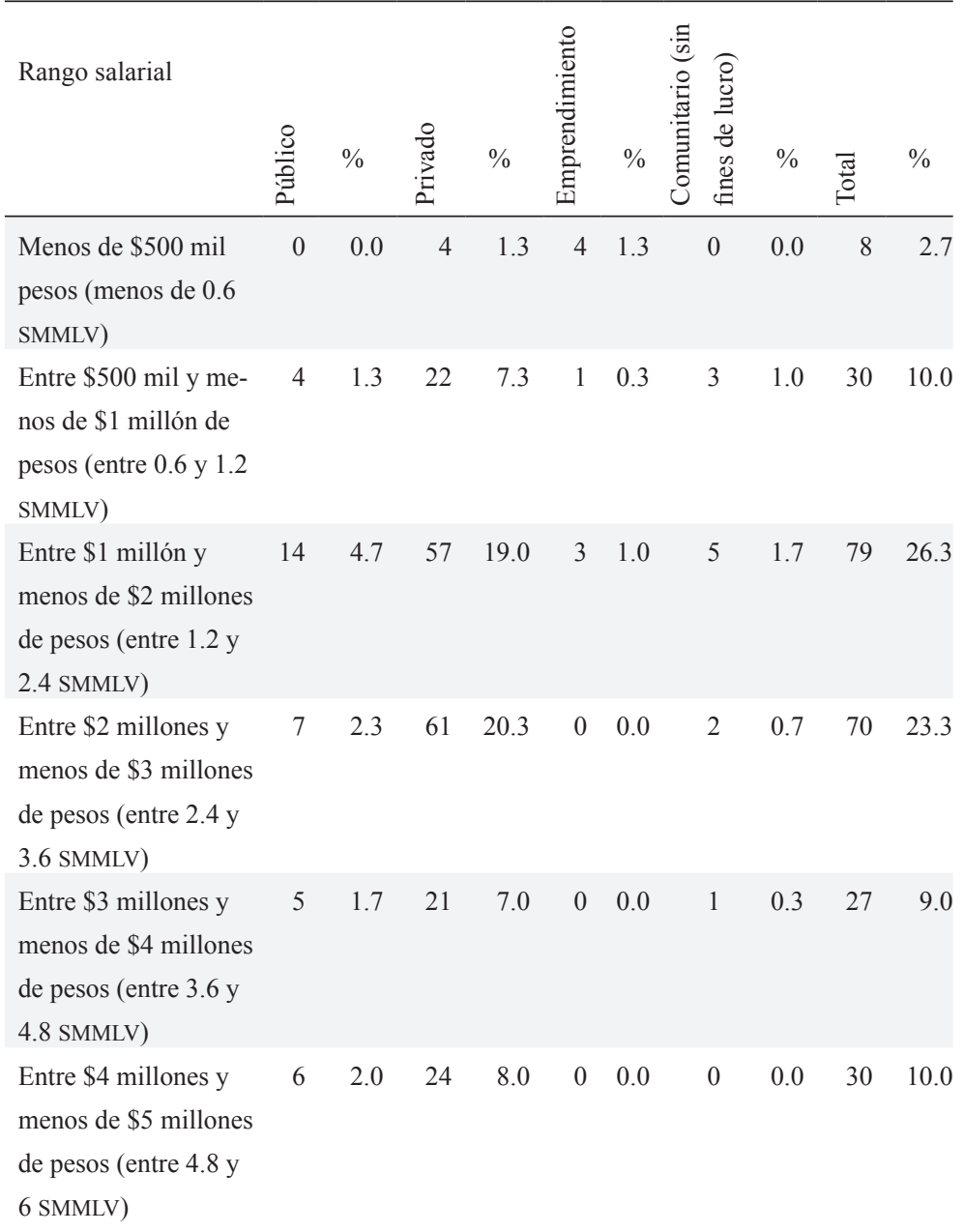




\begin{tabular}{|c|c|c|c|c|c|c|c|c|c|c|c|}
\hline Rango salarial & 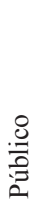 & $\%$ & $\begin{array}{l}\frac{8}{7} \\
\stackrel{2}{2} \\
2\end{array}$ & $\%$ & 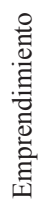 & $\%$ & 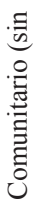 & 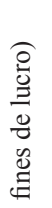 & $\%$ & 胥 & $\%$ \\
\hline $\begin{array}{l}\text { Entre } \$ 5 \text { millones y } \\
\text { menos de } \$ 6 \text { millones } \\
\text { de pesos (entre } 6 \text { y } 7.2 \\
\text { SMMLV) }\end{array}$ & 7 & 2.3 & 15 & 5.0 & 3 & 1.0 & & 1 & 0.3 & 26 & 8.7 \\
\hline $\begin{array}{l}\text { De } \$ 6 \text { millones de } \\
\text { pesos en adelante } \\
\text { (más de } 7.2 \text { SMMLV) }\end{array}$ & 6 & 2.0 & 24 & 8.0 & 0 & 0.0 & & 0 & 0.0 & 30 & 10.0 \\
\hline Total & 49 & 16.3 & 228 & 76.0 & 11 & 3.7 & & 12 & 4.0 & 300 & 100.0 \\
\hline
\end{tabular}

Fuente: Datos de la investigación.

Al comparar los datos de Gutiérrez-Coba et al., (2010) con los de la investigación presente, es posible notar cierta desmejora en el nivel de remuneración de los periodistas colombianos. En 2010 el SMMLV se encontraba en 515 mil pesos colombianos, y el 34\% de los encuestados declaraba ganar entre dos y cuatro SMMLV, mientras el 27.6\% recibía entre uno y dos SMMLV. El 6\% ganaba menos de un salario mínimo y el $32.4 \%$ recibía más de cinco SMMLV. Esto equivalía a decir que el $61.6 \%$ de los periodistas devengaba entre uno y cuatro SMMLV. Para 2019 , el $49.6 \%$ percibía por su trabajo entre 1.2 y $3.6 \mathrm{SMMLV}$, mientras un $12.7 \%$ recibía menos de un salario mínimo. El 28.7\% recibía más de cinco SMMLV (ver Figura 1).

Esta situación resulta más compleja si se tiene en cuenta la devaluación del peso con respecto al dólar. Así, un salario mínimo en 2010 correspondía a alrededor de 269 dólares, mientras en 2019, cuando fueron analizados los datos de la investigación, equivalía a 259 dólares, es decir, 10 dólares menos que hace una década.

La asociación entre nivel salarial y formación educativa (Coeficiente de Contingencia $=0.52 ; p<0.05$ ) se verifica la hipótesis de que, a mayor formación educativa, mayor nivel de ingresos, en más del 50\% de los casos. Por ejemplo, de los 66 periodistas con maestría, el $62 \%$ devenga más de 3.6 SMMLV y de ellos el $22.7 \%$ dice ganar más de 7.2 
SMMLV. Igual ocurre con los 41 que tienen título de especialistas, de quienes el 58.5\% gana más de 3.6 SMMLV. Entre tanto, de los 175 que reportaron tener título de grado universitario, solo el $25.7 \%$ gana más de 3.6 SMMLV (ver Figura 2).

\section{FIGURA 1}

COMPARATIVO SALARIOS PERIODISTAS 2010-2019 (EN PORCENTAJE)

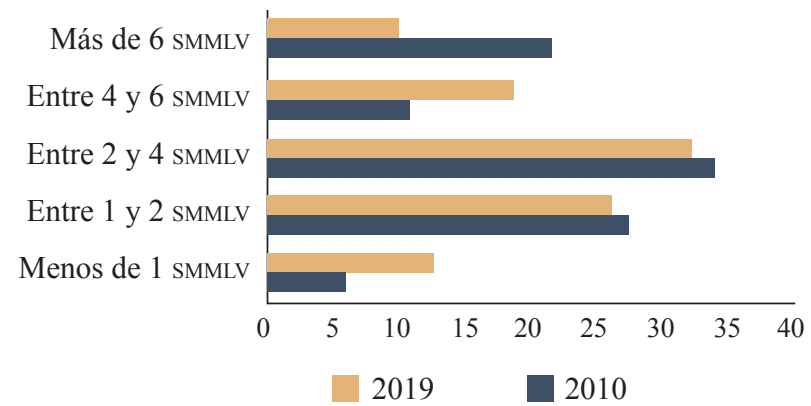

Fuente: Datos de la investigación.

\section{FIGURA 2}

RANGO SALARIAL SEGÚN NIVEL EDUCATIVO

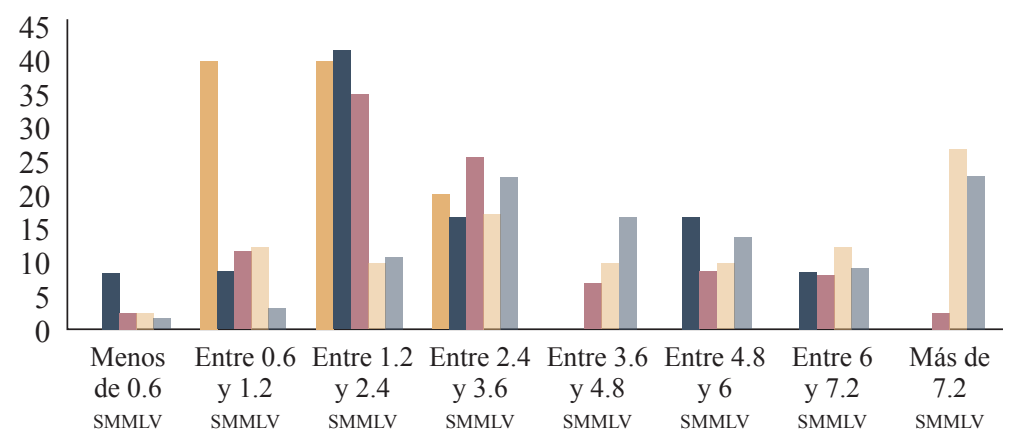

Secundaria $\square$ Técnico $\square$ Universitario $\square$ Especialización $\square$ Maestría

Fuente: Datos de la investigación. 
La relación hipotética entre la experiencia laboral y el salario (Coeficiente de contingencia $=0.55 ; p=0.05$ ) es mediana y significativa. Así, el $57.6 \%$ de los 59 periodistas que llevan más de 20 años de ejercicio profesional recibe más de $3.6 \mathrm{SMMLV}$, al igual que el $60 \%$ de los 40 periodistas que tienen entre 16 y 20 años de actividad laboral y el 54.6\% de los 44 periodistas con experiencia entre 11 y 15 años. En cambio, solo el $32 \%$ de los 53 periodistas que llevan entre seis y diez años de experiencia superan los 3.6 SMMLV y solo llegan a este rango salarial el $13.8 \%$ de los 104 periodistas que tienen menos de cinco años de experiencia laboral.

En cuanto al tipo de contratación, el $47.7 \%$ de los encuestados contaba con contrato a término indefinido, seguido del $23 \%$ que estaba contratado por prestación de servicios y por el $17.7 \%$ que estaba contratado a término fijo. La modalidad de cupos publicitarios, en la que el periodista se ve en la obligación de vender publicidad al mismo tiempo que produce los contenidos informativos, y que era muy frecuente en épocas anteriores en Colombia, aparece en la encuesta con un 3.3\%, lo cual muestra una mejoría en la situación de los informadores (ver Figura 3).

FIGURA 3

TIPO DE VINCULACIÓN LABORAL

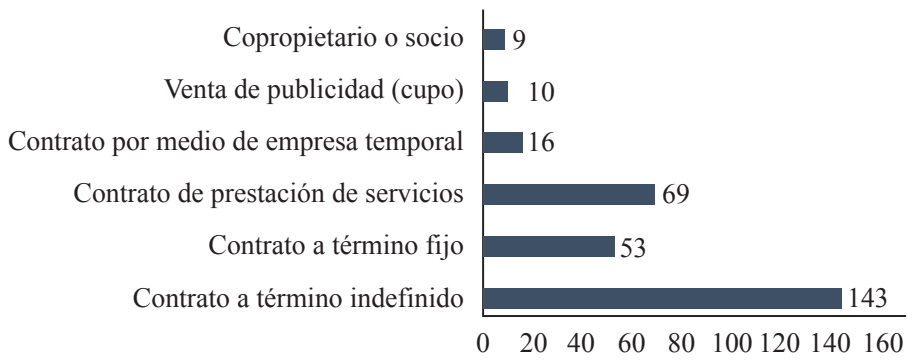

Fuente: Datos de la investigación.

Igualmente, al $66.7 \%$ de los consultados las empresas periodísticas les cubren las prestaciones sociales (pensión, salud, administradora de riesgos laborales, caja de compensación familiar), pero sigue existiendo un $33.3 \%$ que queda desprotegido en estos aspectos. 
Para la mayor parte de los periodistas consultados (64.3\%), la jornada laboral comprende entre ocho y diez horas diarias de trabajo y al $62.3 \%$ no le exigen dedicación exclusiva, de manera que pueden tener varios empleos de manera simultánea.

En las entrevistas se evidencia preocupación por las condiciones laborales, especialmente de los jóvenes, donde ven mucha inestabilidad y el peligro latente de la pérdida de identidad, relacionada con el rol que cumple el periodista en la sociedad como vocero de la comunidad y defensor de la verdad. "Si un trabajo no es bien remunerado, por más pasión que se sienta por la profesión terminará afectando su desempeño, terminando por adoptar la ley del menor esfuerzo y sin importarle la responsabilidad que tiene como puente entre la ciudadanía y la información" (Periodista de radio, Bogotá, entrevista junio 20 de 2018). Otro informador de prensa señala: "El grado de compromiso con la verdad que tiene un periodista que se siente bien en su trabajo es mucho mayor comparado con el que no lo siente" (Periodista de periódico, Bogotá, 18 de mayo de 2018).

Así, por ejemplo, de los 40 entrevistados, los que llevaban más de diez años en la profesión no consideraban cambiar el periodismo por otra actividad y justificaban su decisión en la pasión por lo que hacen y en la dificultad de adaptarse a algo muy distinto a lo que siempre han hecho. Estas personas también decían sentirse a gusto con sus condiciones laborales actuales y su remuneración. Entre tanto, aquellos que tenían menos de diez años en el ejercicio profesional del periodismo declararon que sí cambiarían de actividad: "Lo haría si llega la oportunidad de tener mejores condiciones laborales y salariales" (Periodista de radio, Bogotá, 15 de mayo de 2018); "Sí lo he pensado. Somos seres humanos y tenemos familia y pienso en otras opciones para poder vivir mejor" (Periodista de radio, Bogotá, 10 de junio de 2018); "Yo creo que sí, en el sentido de que si otra profesión u otra actividad me asegura un mejor futuro creo que la tomaría, si me llama la atención, tengo el talento y las capacidades de hacerla. No tengo otra opción clara, pero si se me presenta otra oportunidad mejor seguramente la tomaría" (Periodista de televisión, Bogotá, 12 de junio de 2018). 


\section{Periodistas medianamente satisfechos}

El 51.3\% de los periodistas tiene una percepción de satisfacción laboral global media, seguido de un $30.3 \%$ que tiene una percepción de satisfacción alta (ver Figura 4).

\section{FIGURA 4}

PERCEPCIÓN DE SATISFACCIÓN LABORAL DE LOS PERIODISTAS

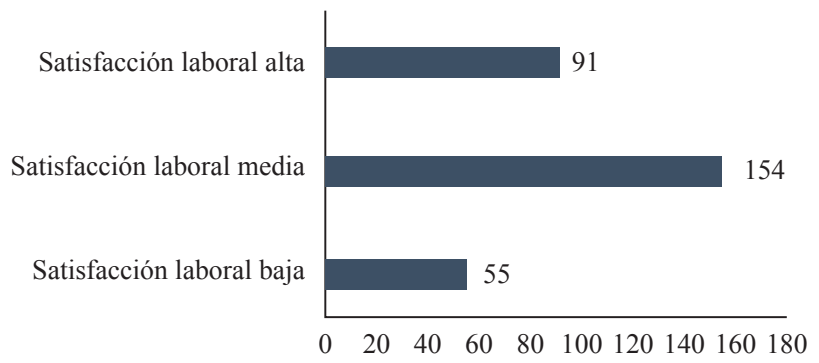

Fuente: Datos de la investigación.

En las entrevistas se evidencia que son los factores relacionados con las motivaciones para realizar el trabajo y la función social del periodismo los que influyen en la buena percepción global de satisfacción. Así lo relata un periodista radial, con nueve años de experiencia profesional: "Lo que más me genera satisfacción es saber que podemos ser la voz de aquellas personas que tienen denuncias importantes, pero no hacen eco hasta que no llegan a los medios" (Periodista de radio, Bogotá, 10 de mayo de 2018). También lo destaca un periodista de un canal público de televisión, con más de diez años en la profesión: "Me satisface servir a los demás y ayudar a muchas personas a mejorar su situación" (Periodista de televisión, Bogotá, 25 de junio de 2018).

Al discriminar las escalas por factores intrínsecos y extrínsecos, se encuentra que la satisfacción laboral intrínseca es alta para el 42.3\% de los encuestados, seguida por el $36.7 \%$ que la ubica en el rango medio. Entre los factores que más influyen en esta percepción positiva están la autonomía para desarrollar una manera propia de trabajo, que es valorada satisfactoriamente por el $63 \%$; el apoyo que reciben de sus je- 
fes directos para realizar bien su trabajo, con un 59\% de favorabilidad, y el estímulo que les expresan sus jefes cuando realizan bien su labor, que es bien valorado en un $42 \%$ de los casos.

Sin embargo, el 51\% está insatisfecho con las posibilidades de ascenso o promoción que tienen en su trabajo. Esto sale a la luz en las entrevistas, como lo relata un periodista web, con siete años de trayectoria: "Me molesta que sea tan difícil progresar en la carrera, porque el medio no es tan amplio, hay muchas dificultades de ejercer y ascender" (Periodista de medio digital, Bogotá, 15 de agosto de 2018).

En cuanto a la satisfacción laboral extrínseca, el $49 \%$ de los encuestados la califica en el rango medio, seguido de un $29.7 \%$ que la califica en el nivel alto. Entre los factores considerados en esta escala que llaman la atención se encuentra que el 31\% de los periodistas se siente poco o nada satisfecho con el salario, frente a un $36 \%$ que se siente bastante o muy satisfecho y un 33\% medianamente satisfecho. Un periodista de un medio impreso con más de diez años de experiencia laboral expresa: "En Colombia el trabajo del periodista es bastante riesgoso y no tiene una valoración salarial acorde con el peligro que implica" (Periodista de revista, Bogotá, 5 de julio de 2018).

Entre los factores que generan mayor percepción positiva en la satisfacción extrínseca se encuentran las condiciones físicas del lugar de trabajo, bien valoradas por el $64 \%$ de los encuestados; las condiciones de salud ocupacional y prevención de accidentes, con las que está satisfecho el 55.3\%; y el horario, con el cual se siente satisfecho el $47.3 \%$ de los periodistas consultados.

En cambio, son factores de insatisfacción los cursos de capacitación o actualización que ofrece la empresa, con los cuales está poco o nada satisfecho el 42.3\%; los programas de desarrollo profesional, con los cuales está insatisfecho el 39.7\%, y las actividades de bienestar que ofrece la empresa para la familia del trabajador, que generan insatisfacción en el $56.7 \%$ de los periodistas. En las entrevistas, el testimonio de un periodista relata: "Trabajo para dos medios de comunicación y no hay apoyo para la capacitación. Actualmente adelanto mi carrera profesional, pero todo corre por mi cuenta, y en lo que se refiere a cursos de nuevas plataformas digitales, son muy esporádicos" (Periodista de canal regional de televisión, Cali, 9 de mayo de 2018). 
Como se observa, los factores extrínsecos contribuyen a que la percepción de satisfacción laboral baje en los periodistas encuestados (ver Figura 5).

FIGURA 5

PERCEPCIÓN DE SATISFACCIÓN SEGÚN

FACTORES INTRÍNSECOS Y EXTRÍNSECOS

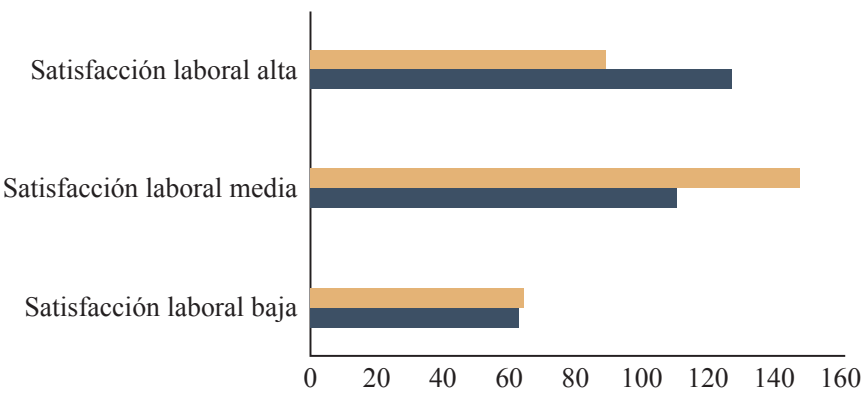

Factores extrínsecos $\square$ Factores intrínsecos

Fuente: Datos de la investigación.

\section{Cómo influye el contexto de país en el trabajo de los periodistas}

Las entrevistas sacaron a la luz ciertos aspectos que no estaban contemplados en la encuesta y que son generadores de insatisfacción entre los periodistas colombianos, entre ellos, el nuevo panorama empresarial generado por las Tecnologías de la Información y la Comunicación, TIC's, y las presiones a las que se ven sometidos por diferentes actores.

Los periodistas entrevistados señalan que las tecnologías han cambiado la manera de acceder a la información y que han traído consigo cierto facilismo, del cual ellos mismos serían directamente responsables: "Me molesta la pereza de los colegas. No saben lo que tienen entre manos. No llaman, no investigan, no salen a buscar información" (Periodista de emprendimiento digital, Bogotá, 11 de julio de 2018).

Sin embargo, también responsabilizan a las empresas periodísticas que, en el afán por superar la crisis que enfrenta el modelo de negocio 
financiado por la pauta comercial, han optado por disminuir la planta de reporteros, rebajar las inversiones en reportería directa y buscar tráfico en las redes sociales. En ese contexto, los periodistas sienten que realizan más tareas que antes, como explica un reportero: "Nosotros nos encargamos de redactar el artículo, editar el video, somos multidisciplinarios porque somos editores, redactores, diseñadores y distribuidores" (Periodista de canal de televisión local, Medellín, 3 de mayo de 2018).

Por otra parte, muchos reiteran que se sienten presionados, directa o indirectamente, por las relaciones que los medios tienen con el Gobierno o con grupos económicos particulares. "A veces, por ciertos compromisos del medio donde se trabaja, uno no puede desenvolverse libremente" (Periodista de canal de televisión regional, Bucaramanga, 20 de mayo de 2018). Otro periodista de un medio radial, de carácter privado, señala: "Los medios responden hacia determinada ideología política o hacia ciertas relaciones de amistad y a veces uno se siente amarrado" (Periodista de radio, Bogotá, 11 de mayo de 2018).

Y aunque ninguno de los entrevistados sufría actualmente de amenazas por parte de actores fuera de la ley, reconocen que existen colegas que deben enfrentar estas situaciones, como explica un periodista de un medio radial comunitario: "En Bogotá hay un poco más de libertad para desarrollar el ejercicio periodístico, pero en otras regiones no está esa posibilidad, porque estamos en un país donde todavía matan periodistas" (Periodista de radio comunitaria, Bogotá, 5 de agosto de 2018).

\section{CONCLUSIONES}

Si bien los resultados de esta investigación no pueden generalizarse para toda la población de periodistas colombianos, ya que se desconoce su número exacto, la muestra de 300 periodistas, de 123 medios de comunicación en 24 ciudades del país, permite dar una idea de la situación que muchos de ellos viven. El panorama laboral de los periodistas colombianos sigue siendo preocupante diez años después del estudio más reciente sobre el tema (Gutiérrez-Coba et al., 2010). El nivel salarial ha desmejorado en términos del número de Salarios Mínimos Mensuales Legales Vigentes que recibe un periodista por su labor, sobre todo en los rangos de remuneración más altos, aunque ha aumentado en los rangos bajos. 
Por otra parte, si bien los salarios han empeorado, las condiciones de contratación parecen haber mejorado, ya que la mayoría cuenta con contratos a término indefinido y son pocos los que se enfrentan a una práctica que durante años fue recurrente en las regiones y comprometía la libertad de prensa: la venta de cupos publicitarios. También es positivo que las empresas estén cumpliendo mayoritariamente con la obligación de cubrir las prestaciones sociales de los periodistas, pero hay aún un $33.3 \%$ que sigue estando desprotegido y se requeriría una intervención de los gremios de periodistas y del propio Gobierno para dar solución a estos casos.

Sin embargo, más que con la remuneración salarial, los periodistas se sienten inconformes con la falta de oportunidades de ascenso en su trabajo, y con la falta de apoyo para seguir estudiando de manera formal (especializaciones o maestrías) o para capacitarse a través de cursos cortos o talleres.

Esto resulta de especial importancia, ya que se observa que quienes están mejor remunerados cuentan en su mayoría con formación postgradual, lo cual habla de la necesidad de que los periodistas sigan estudiando después de terminada la carrera universitaria, para lo cual requieren apoyo en tiempo y dinero por parte de las empresas para las cuales trabajan. Sin embargo, es necesario reconocer que el número de periodistas con postgrado se ha triplicado en comparación a lo que sucedía diez años atrás, lo cual puede deberse a la existencia de una mayor oferta de programas de este tipo en el país, y también a un creciente interés de los periodistas por cualificarse.

En las regiones, los informadores claman por mayor capacitación y el tema resulta bastante sensible si se tiene en cuenta que las TIC han llevado a que el periodista deba trabajar para distintas plataformas de manera simultánea, muchas veces sin tener los conocimientos necesarios para hacerlo. Aquí el trabajo de los gremios de periodistas puede ser importante, toda vez que pueden gestionar cursos cortos que sean impartidos en algunas ciudades intermedias.

Por la población que respondió la encuesta, se podría inferir que las salas de redacción de los medios colombianos están conformadas mayoritariamente por hombres, lo que daría pie a una investigación sobre la posible existencia de una brecha de género en este campo laboral. En 
cambio, resulta positivo ver que existe una adecuada distribución de profesionales con poca, media y larga trayectoria profesional, ya que la experiencia de los que más tiempo llevan puede enriquecer a quienes llevan menos años en la labor informativa que, por su parte, pueden alimentar con nuevas ideas y manejo de herramientas a quienes llevan más años en la profesión.

En cuanto a los niveles de satisfacción con su labor, los periodistas tienen una percepción global de satisfacción media. Sin embargo, cuando se observan por separado los factores intrínsecos, dependientes de sus propias motivaciones, y los extrínsecos, dependientes de factores externos, se encuentran marcadas diferencias. La satisfacción intrínseca es mayoritariamente alta, mientras que la extrínseca es mayoritariamente media.

La satisfacción intrínseca es alta porque la autonomía en el trabajo y los valores tradicionales del periodismo, como dar voz a quienes no la tienen y servir a la comunidad, continúan siendo los principales motivadores para los informadores, quienes además valoran mucho recibir apoyo de sus jefes para realizar su propia forma de trabajo y se sienten satisfechos cuando los jefes resaltan que han realizado un buen trabajo, como también ha sido registrado en investigaciones precedentes en otros países (Rodríguez, 2003; Weaver \& Wilhoit, 1996). Aun así, los periodistas dejan ver su inconformidad con el condicionamiento que representa para ellos saber que la propiedad del medio está en manos de un grupo económico con intereses particulares o de los gobiernos locales o regionales porque, aunque no exista censura directa, los periodistas tienden a evitar ciertos temas que afecten los intereses de los grupos de poder a los que están adscritos los medios para los cuales trabajan y advierten que sienten coartada su libertad de expresión.

En cuanto a la satisfacción extrínseca, mayoritariamente media, es fácil identificar aspectos en los cuales las empresas informativas podrían generar cambios concretos y sencillos para mejorar la percepción de satisfacción. Organizar cursos de actualización y entrenamiento en habilidades específicas y manejo de herramientas digitales, realizar actividades de bienestar para las familias de los periodistas, apoyar la formación de los periodistas otorgando tiempos para que estudien postgrados, generar mayores posibilidades de ascenso a cargos de respon- 
sabilidad, son algunas de las acciones posibles. Esto no solo mejoraría la percepción de satisfacción por su trabajo, sino que terminaría dando mejores réditos en lo que se refiere al compromiso laboral y a la estabilidad.

Por otra parte, las malas condiciones laborales, especialmente de los periodistas más jóvenes, parecen afectar directamente a su motivación para realizar bien el trabajo y a su identidad profesional. Prueba de ello es que, si les dieran posibilidades mejores, cambiarían de actividad laboral sin problema. Esto último va en detrimento de la calidad del periodismo y es un tema en el que se debe profundizar, porque la alta rotación de periodistas en los medios es otro de los problemas que influye en la calidad de la información que se produce, aún con la posibilidad que hoy brindan las tecnologías de acceder directamente a la información sin intermediación alguna, los medios de comunicación siguen siendo necesarios como fuentes confiables, de manera que lo que ocurra con los profesionales que los alimentan, debe ser tema de interés para toda la sociedad.

Si bien la calidad del periodismo que reciben los ciudadanos no depende solo de la situación laboral y la satisfacción de los periodistas con su trabajo, es uno de los factores que más influyen en ello y es necesario que tanto empresas periodísticas como gremios profesionales presten atención a ello.

Agradecimientos a los auxiliares de investigación del Grupo de Investigación en Periodismo: Juan David Botero, Nicolás Medellín, Sebastián Vallejo, Camilo Páez y Javier Saba.

\section{Referencias bibliográficas}

Arroyave, J. \& Blanco, I. (2005). Cómo perciben los periodistas su profesión: Entre el agotamiento y la fascinación. Investigación \& Desarrollo, 13(2), 364-389. http://rcientificas.uninorte.edu.co/index.php/ investigacion/article/view/1004/630

Barragán, C. (2018). III Estudio de medios digitales en Colombia - La Babel Digital. https://consejoderedaccion.org/webs/lababeldigital/ contenidos/iii-estudio-de-medios-digitales-en-colombia/ 
Barrios, A. \& Zambrano, W. (2015). Convergencia Digital: Nuevos perfiles profesionales del periodista. Anagramas - Rumbos y sentidos de la comunicación, 13(26), 221-240. https://doi.org/10.22395/ angr.v13n26a11

Barrios, M. M. \& Arroyave, J. (2007). Perfil Sociológico de la profesión del periodista en Colombia: Diálogo íntimo con el ser humano detrás de las noticias. Diálogos de la Comunicación, 75, 1-15. http://www.academia.edu/download/34356868/Perfil_sociologico_ de_la_profesion_del_periodista_en_Colombia_-_Barrios_y_Arroyave.pdf

Becerra, M. \& Mastrini, G. (2017). Concentración y convergencia de medios en América Latina. Communiquer. Revue de communication sociale et publique, 20, 104-120. https://doi.org/10.4000/communiquer.2277

Bowling, N. A., Khazon, S., Meyer, R. \& Burrus, C. J. (2015). Situational Strength as a Moderator of the Relationship Between Job Satisfaction and Job Performance: A Meta-Analytic Examination. Journal of Business and Psychology, 30(1), 89-104. https://oi. org/10.1007/s10869-013-9340-7

Cantalapiedra, M. J., Coca, C. \& Bezunartea, O. (2016). La situación Profesional y laboral de los periodistas vascos. ZER: Revista de Estudios de Comunicación, 5(9). https://www.ehu.eus/ojs/index.php/ Zer/article/view/17441

Casero-Ripollés, A. \& Rabadán, P. L. (2013). La gestión de fuentes informativas como criterio de calidad profesional. En J. L. Gómez Mompart, J. F. Gutiérrez Lozano \& D. Palau Sampio (Eds.), La Calidad periodística: teorías, investigaciones y sugerencias profesionales (pp. 73-89). Aldea Global. https://dialnet.unirioja.es/servlet/ articulo? codigo $=5267178$

Consejo de Redacción \& Poderopedia. (2015). Mapa de Medios. Influence Mapping Group. https://www.influencemapping.org/projects/mapa-de-medios/

Díaz Jordán, A. M. \& Serrano, N. (2017). La profesión periodística en el caribe colombiano: Entre percepción y significados. Investigación \& Desarrollo, 25(1), 73-101. https://doi.org/10.14482/i\&d. v25i1.8630 
Espino, G. (2016). Periodistas precarios en el interior de la república mexicana: Atrapados entre las fuerzas del mercado y las presiones de los gobiernos estatales. Revista Mexicana de Ciencias Politicas y Sociales, 61(228), 91-120. http://dx.doi.org/10.1016/S01851918(16)30041-1

Farías, P. \& Gómez, M. (2011). El estado de la profesión periodística y la crisis de los medios en España. Razón y Palabra, 16(77). http:// www.razonypalabra.org.mx/varia/77\%205a\%20parte/68_FariasGomez_V77.pdf

Federación Colombiana de Periodistas-Fecolper. \& Reporteros Sin Fronteras. (s. f.). Media Ownership Monitor Colombia. Media Ownership Monitor. https:/colombia.mom-rsf.org/es/

Fisher, C. D. (2000). Mood and Emotions while Working: Missing Pieces of Job Satisfaction? Journal of Organizational Behavior, 21(2), 185-202. https://doi.org/10.1002/(SICI)1099-13 79(200003)21:2<185::AID-JOB34>3.0.CO;2-M

Fritzsche, B. A. \& Parrish, T. J. (2005). Theories and Research on Job Satisfaction. En S. D. Brown \& R. W. Lent (Eds.), Career development and counseling: Putting theory and research to work (pp. 180-202). John Wiley \& Sons Inc.

Fundación para la Libertad de Prensa-FLIP. (s. f.). Cartografías de la Información. https://flip.org.co/cartografias-informacion/

Fundación para la Libertad de Prensa-FLIP. (2018). Informe anual: Prensa acorralada: un juego de violentos y poderosos. https://flip. org.co/index.php/es/publicaciones/informes-anuales/item/2315-informe-anual-prensa-acorralada-un-juego-de-violentos-y-poderosos

Garcés, M. \& Arroyave, J. (2017). Autonomía profesional y riesgos de seguridad de los periodistas en Colombia. Revista Perfiles Latinoamericanos, 25(49), 35-53. https://doi.org/10.18504/pl2549-0022017

Gómez-Mompart, J., Gutiérrez-Lozano, J. \& Palau-Sampio, D. (2013). La Calidad periodística teorías, investigaciones y sugerencias profesionales. Universidad de Barcelona.

Gutiérrez-Coba, L. (2009). Análisis de la calidad informativa, primer paso hacia el cambio. Palabra Clave, 9(1). https://palabraclave.unisabana.edu.co/index.php/palabraclave/article/view/1227 
Gutiérrez Coba, L., Prada Penagos, R., Valderrama Valderrama, J., García Perdomo, V., Guzmán de Reyes, A. \& Forero Gutiérrez, A. (2010). Las condiciones laborales y la satisfacción de los periodistas colombianos. Investigación \& Desarrollo, 18(1). https://doi. org/10.14482/i\&d.v18i1.965

Huerta-Wong, J. E. \& García, R. G. (2013). Concentración y diversidad de los medios de comunicación y las telecomunicaciones en México. Comunicación y Sociedad, 19, 113-152. http://www.comunicacionysociedad.cucsh.udg.mx/index.php/comsoc/article/view/206 International Telecommunication Union. (2018). Measuring the Information Society Report 2018. International Telecommunication Union. https://www.itu.int/en/ITU-D/Statistics/Pages/publications/ misr2018.aspx

Manrique, A. \& Cardona, I. (2003). Situación laboral de los periodistas en Colombia. Informe de la investigación. Antropos (Cátedra Konrad Adenauer de Comunicación y Democracia).

Márquez-Ramírez, M. \& Hughes, S. (2017). Panorama de los perfiles demográficos, laborales y profesionales de los periodistas en México: Reporte de Investigación. Global Media Journal México, 14(26). https://journals.tdl.org/gmjei/index.php/GMJ_EI/article/vi ew/281

Mellado, C. \& Lagos, C. (2013). Características y factores condicionantes de la situación salarial de los periodistas en Chile. Palabra Clave, 16(2), 520-540. https://doi.org/10.5294/pacla.2013.16.2.10

Mellado, C. \& Parra, E. (2008). Indicadores de identidad y perfil del periodista regional en Chile. Opción: Revista de Ciencias Humanas y Sociales, 55, 145-167. http://ve.scielo.org/scielo.php?script=sci_art text\&pid=S1012-15872008000100009

Meyer, R. D., Dalal, R. S. \& Hermida, R. (2010). A Review and Synthesis of Situational Strength in the Organizational Sciences. Journal of Management, 36(1), 121-140. https://doi.org/10. 1177/0149206309349309

Ministerio de Tecnologías de la Información y las Comunicaciones. (2019). Boletín trimestral de las TIC. Cifras Cuarto Trimestre de 2018. https://colombiatic.mintic.gov.co/679/w3-article-100444.html

Odriozola, J., Aguirre, C. \& Roa, M. S. (2016). Los condicionantes en el desarrollo de una buena praxis periodística en Ecuador: Estudio 
de los factores de influencia según los profesionales ecuatorianos. Revista ComHumanitas, 7(Extra 1), 89-109. http://www.comhumanitas.org/index.php/comhumanitas/article/view/Odriozola

Pellegrini, S., Puente, S., Porath, W. \& Mujica, C. (2011). Valor agregado periodístico. La apuesta por la calidad de las noticias. Universidad Católica de Chile.

Rey, G. \& Novoa, J. L. (2012). Medios digitales en Colombia. Agendas periodisticas y sostenibilidad. Universidad Javeriana, Consejo de Redacción. http://consejoderedaccion.org/webs/documentos/Estudio_medios_digitales_2012.pdf

Reyes, A. R., González, A. E., Torrijos, J. L. R. \& Gordillo, M. del M. G. (2015). La calidad de los medios y el uso de fuentes periodísticas en la prensa local de referencia en España. Estudios sobre el Mensaje Periodístico, 21, 85-100. https://doi.org/10.5209/rev_ ESMP.2015.v21.50659

Rodríguez, R. (2003). Características socio-demográficas y laborales de los periodistas españoles e índice de satisfacción profesional. Ámbitos. Revista Internacional de Comunicación, 0(9-10), 487504. https://doi.org/10.12795/Ambitos.2002-2003.i09-10.26

Romero-Rodríguez, L. M., de-Casas-Moreno, P. \& Torres-Toukoumidis, Á. (2016). Dimensiones e indicadores de la calidad informativa en los medios digitales. Comunicar, XXIV(49), 91-100. https://doi. org/10.3916/C49-2016-09

Sabés, F. \& Verón, J. J. (2012). Universidad y empresa ante la doble crisis del periodismo tradicional. Propuestas y reflexiones sobre la modificación sustancial del escenario periodístico. El clúster periodístico. adComunica, 0(4), 151-170-170. https://doi.org/10.6035/74

Schulz, W. (2000). Preconditions of Journalistic Quality in an Open Society. Paper for Presentation at the International Conference News Media and Politics-Independent Journalism. Budapest, 6 y 7 de octubre de 2000. https://www.europesociety.hu/en/blog/preconditions-journalistic-quality-open-society

Thompson, E. R. \& Phua, F. T. T. (2012). A Brief Index of Affective Job Satisfaction. Group \& Organization Management, 37(3), 275-307. https://doi.org/10.1177/1059601111434201 
Trejo, R. (2010). Muchos medios en pocas manos: Concentración televisiva y democracia en América Latina. Intercom. Revista Brasileira de Ciências da Comunicação, 33(1), 17-51. http://portcom.intercom.org. br/revistas/index.php/revistaintercom/article/viewArticle/146

Túñez, M. \& Martínez, M. Y. (2015). Análisis del impacto de la función, las actitudes y las condiciones laborales del periodista en la producción de noticias: Hacia un periodismo de empresa. ZER: Revista de Estudios de Comunicación, 19(36). https://www.ehu.eus/ ojs/index.php/Zer/article/view/13486

Ufarte, M. J. (2012). La situación laboral del periodista como factor condicionante de la calidad informativa: Con precariedad no hay calidad. Actas IV Congreso Internacional Latina de Comunicación Social: comunicación, control y resistencias. https://dialnet.unirioja.es/servlet/articulo? codigo $=4222723$

Weaver, D. H. \& Wilhoit, G. C. (1996). The American Journalist in the 1990s: U.S. News People at the End of an Era. Psychology Press.

Weiss, H. M. (2002). Deconstructing job satisfaction: Separating evaluations, beliefs and affective experiences. Human Resource Management Review, 12(2), 173-194. https://doi.org/10.1016/ S1053-4822(02)00045-1 